TP Periodica Polytechnica Mechanical Engineering

\author{
58(2), pp. 119-126, 2014 \\ DOI:10.3311/PPme.7057 \\ Creative Commons Attribution (1)
}

RESEARCH ARTICLE

\section{The steel damageability simulation under random loading by the power, energetical and strain fracture criterions}

Alexey Nikolaevich Savkin, Alexander Alexandrovich Sedov, Artem Valerievich Andronik

RECeIVEd 02 October 2013; Accepted After ReVISIon 22 April 2014

\begin{abstract}
Effort to describe effect of variable amplitude loading character on fatigue damage cumulation is committed for a metal material of a structural part. It is proposed to quantify the influence of loading character on durability life via damage model incorporating the nonstationarity factor and the spectrum fullness factor. Experimental evidence and analytical results of the proposed model are correlated.
\end{abstract}

\section{Keywords}

spectrum loading ' damage model ' nonstationarity factor . spectrum fullness factor

\footnotetext{
Alexey Nikolaevich Savkin

Automobile Department, Volgograd State Technical University,

Volgograd, Russia

email: txpic@vstu.ru
}

\section{Alexander Alexandrovich Sedov}

Automobile Department, Volgograd State Technical University,

Volgograd, Russia

email: alexander.a.sedov@gmail.com

\section{Artem Valerievich Andronik}

Automobile Department, Volgograd State Technical University,

Volgograd, Russia

email: tank_leclerc@mail.ru

\section{Introduction}

Sufficient reliability of design and minimal essential safety reserve are two permanently desired engineering aims. This is particularly significant for such high risk systems with crucial role of strength-weight ratio as transport systems. Design error in balancing between these two beacons fraught with both unrecoverable structural fracture and hazard for people lives. Therefore, scientists and engineers already revealed several approaches and incessantly continue their academic pursuits for prevention of fallouts. Several effective damage models are analyzed below.

Present article purposed to analyze durability life calculations of hourglass shaped steel specimens on basis of various fracture criterions and to compare analytical and experimental results for random loading.

Low cycle fatigue processes are complex and diversified in metals, therefore plenty factors such as loading, material properties, geometry of parts, environment, etc. have effects from one another and on fatigue in whole. It is a common practice to apply parameter of mechanical or physical nature named as fracture criterion to describe local fracture. Its parameter value changes during appropriate loading and its limit corresponds to fracture point. These days many scientists engaged in durability prediction and damage models development to outline behavior of materials and structures under random loading [1-3].

\section{Material and research method}

Tests were competed on BISS-100 servohydraulic machine [4]. Test frequency was variable and relevant to loading rate $60 \mathrm{kN}$ per second, but this condition was mandatory for all test series. Hourglass shaped specimens (fig. 1) [5] were manufactured of steel GOST 40X (AISI 5140). Mechanical properties and chemical composition of this grade are given in table 1 .

First test series of steel specimens was completed for different, but constant amplitude loading (hereafter referred to as CAL) during each test. Durability life resulted in range of $5 \cdot 10^{3}-10^{7}$ cycles and fatigue curve parameters were defined during the tests. Mechanical hysteresis loops were tracked 
Tab. 1. Mechanical properties and chemical composition of 40X (AISI 5140) steel

\begin{tabular}{|c|c|c|c|c|c|c|c|c|c|}
\hline \multicolumn{10}{|c|}{ Characteristics of mechanical properties } \\
\hline$\sigma_{u}, M P a$ & $\sigma_{f}^{\prime}, M P a$ & $K^{\prime}, M P a$ & $\varepsilon_{f}^{\prime}$ & $b$ & $n^{\prime}$ & $c$ & $\sigma_{-1}, M P a$ & $N_{G}$ & $m$ \\
\hline 976 & 1590 & 1434 & 0.66 & -0.075 & 0.14 & -0.59 & 390 & $8 \cdot 10^{5}$ & 11.8 \\
\hline \multicolumn{10}{|c|}{ Components of chemical composition, $\%$} \\
\hline C & $\mathrm{Si}$ & $\mathrm{Mn}$ & & & $\mathrm{Ni},(\max )$ & $\mathrm{Cu},(\max )$ & & & $\begin{array}{c}P \\
(\max )\end{array}$ \\
\hline $0.36-0.44$ & $0.17-0.37$ & $0.5-0.8$ & & & 0.3 & 0.3 & & & 0.035 \\
\hline
\end{tabular}

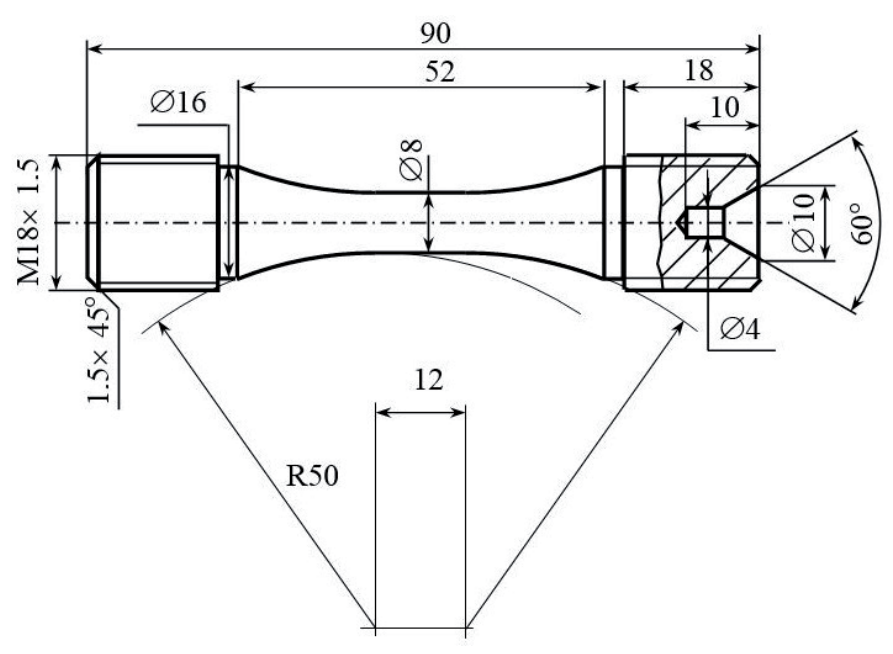

Fig. 1. Drawing of hourglass shaped specimen

during all load histories cycles through extensometer. Width of loop $\Delta \varepsilon_{\text {st }}$ that corresponds to half durability life to fracture $N / 2$ was assumed to be a criterional parameter. Then variable amplitude loading (hereafter referred to as VAL) tests were carried out. All spectrums were processed by rainflow method and transformed to symmetric loading type after Goodman's mean stress correction. Then loading sequences were scaled within the range of -1 to 1 . Thereafter amplitudes of such normalized loading cases were multiplied to specified maximal stress value $\sigma_{a \max }$. Finally, these loading sequences were uploaded into testmanaging program. Extensometer provided feedback signal and tracked inelastic processes kinetics in metal during test as stated above.

\section{Research results}

It is a common knowledge that multiple damage criterions may be classified into several groups [6]: power, strain and energetical criterions. Take a detailed look at material durability according to various low-cycle fatigue criterions under random loading.

\section{Evaluation of fatigue life under spectrum loading via power damage criterions}

Quantification of uncertainty state in spectrum gives a better understanding of loading character influence on fatigue live of structure that undergoes VAL.

It is possible to estimate spectrum loading character through the spectrum fullness factor $V$ by the formula [7]:

$$
V=\left[\frac{1}{v_{b}}\left[\sum_{i=1}^{v_{b}}\left(\frac{\sigma_{a i}}{\sigma_{a \max }}\right)^{m}\right]\right]^{\frac{1}{m}}
$$

where $v_{b}$ - duration (number of cycles) of loading block; $\sigma_{a i}, \sigma_{a \max }-$ values of $i$-th and maximal stress amplitude respectively; $m$ - fatigue curve slope in log-log coordinates.

Actually choice of block duration $V_{b}$ is stipulated by important stages of service loading. In other words, it is essential to meet load altering for different road types at suggested ratios for current service regime. Here are no limitations of block duration value from a mathematical standpoint.

Spectrum generation is a persistently discussing item in scientific community, especially if loading is intended for testing of various materials and structures or simulation. There are presented a lot of different approaches and concepts in the recent papers. For example, authors [8, 9] use the autocorrelation function of VAL for stress sequence to estimate spectrum irregularity. Autocorrelation is the cross-correlation function of external signal in different time points. The Rayleigh distribution is suited enough for the various real service loadings, and hence it was reasonably chosen for present research. Loading simulation is based on that components of two-dimensional vector are independent and distribute random variables according to the Rayleigh distribution. Three loading spectrums were generated via autocorrelation approach on condition that duration of each spectrum is 5000 cycles. This block duration is typical for all VAL and simplifies comparison. Recall that all spectrums were processed by rainflow method and transformed to symmetric loading. Automobile service spectrums SAESUS, SAEBRACKET and SAETRANS were also subjected to this procedure for subsequent comparison of generated sequences 
and well-known spectrums. Some spectrums properties are shown in table 2. Several examples of generated spectrums are demonstrated in fig. 2.

Tab. 2. Properties of the normalized loading spectrums

\begin{tabular}{ccc}
\hline $\begin{array}{c}\text { Loading } \\
\text { spectrum }\end{array}$ & $\begin{array}{c}\text { Spectrum fullness } \\
\text { factor } V\end{array}$ & Block loading durability $V_{b}$ \\
\hline CAL & 1 & $N_{i}$ \\
Spectra A & 0.676 & 5000 \\
Spectra B & 0.591 & 5000 \\
Spectra C & 0.52 & 5000 \\
SAESUS & 0.511 & 2484 \\
SAEBRACKET & 0.524 & 2496 \\
SAETRANS & 0.566 & 1494 \\
\hline
\end{tabular}

It is well known that fatigue curve follows the equality:

$$
N=N_{G}\left(\frac{\sigma_{-1}}{\sigma_{a \max }}\right)^{m},
$$

where values $\sigma_{-1}, m, N_{G}$ are shown in table 1 .

Total fatigue life can be determined by expression:

$$
N_{\Sigma}=N \cdot K
$$
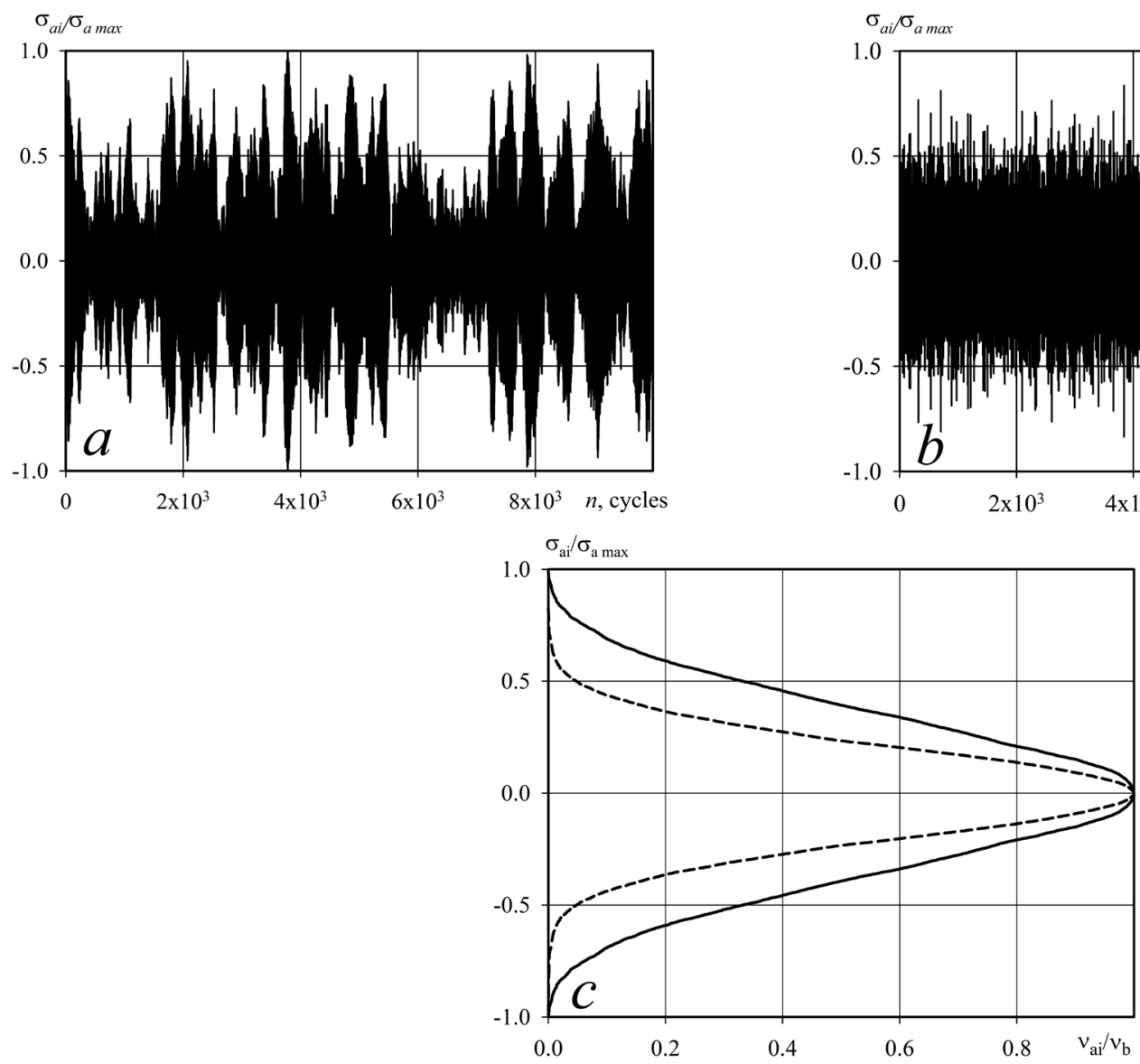

Fig. 2. Examples of loading spectrums: a - block of the spectrum $\mathrm{A}(V=0.676)$; $\mathrm{b}$ - block of the spectrum $\mathrm{C}(V=0.52)$; $\mathrm{c}$ - processed spectrums (- spectra A, - spectra C) where $N$ - fatigue life under CAL; $K$ - nonstationarity factor of loading spectrum.

Resulting nonstationarity factors of spectrums $K$ for various models are summarized in table $3 . K$ values were evaluated by the linear model [10], corrected linear model [11], and generalized model for random loading of steel [12]:

$$
N_{\Sigma}=\frac{N_{G} \cdot 10^{(1+q \lg m)(1-V)}}{\left(\frac{\sigma_{a \max }}{\sigma_{-1}}\right)^{m}}
$$

where $q$ is material constant and $q=3.83$ for steels.

Received empirical fatigue curves for AISI 5140 are presented in fig. 3a for CAL (curve a), spectrum A (dotted $\bullet$ ) and spectrum $\mathrm{C}$ (dotted $\boldsymbol{\Delta}$ ). Curves b and c were drawn on the basis of equation (3) considering most appropriating nonstationarity factor K3 for various spectrum loading.

Calculation results of durability life are shown in table 4 appropriating to different damage models and loading spectrums with $\sigma_{a \max }=650 \mathrm{MPa}$ for steel AISI 5140 .

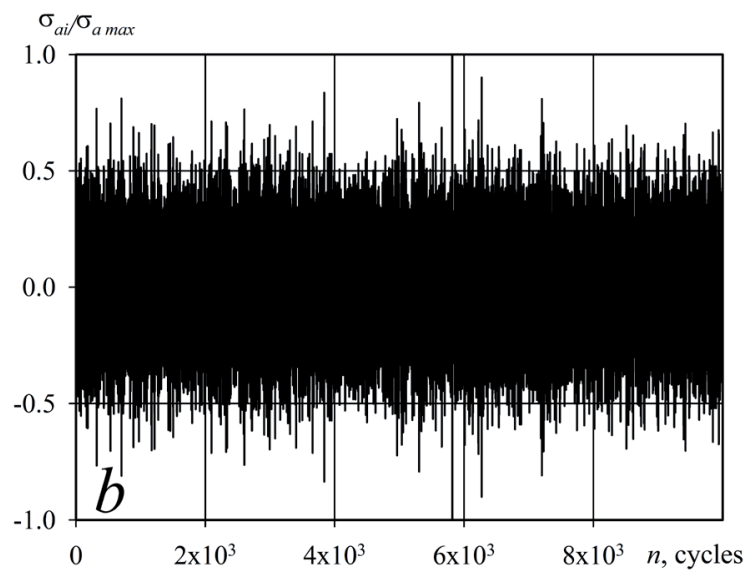



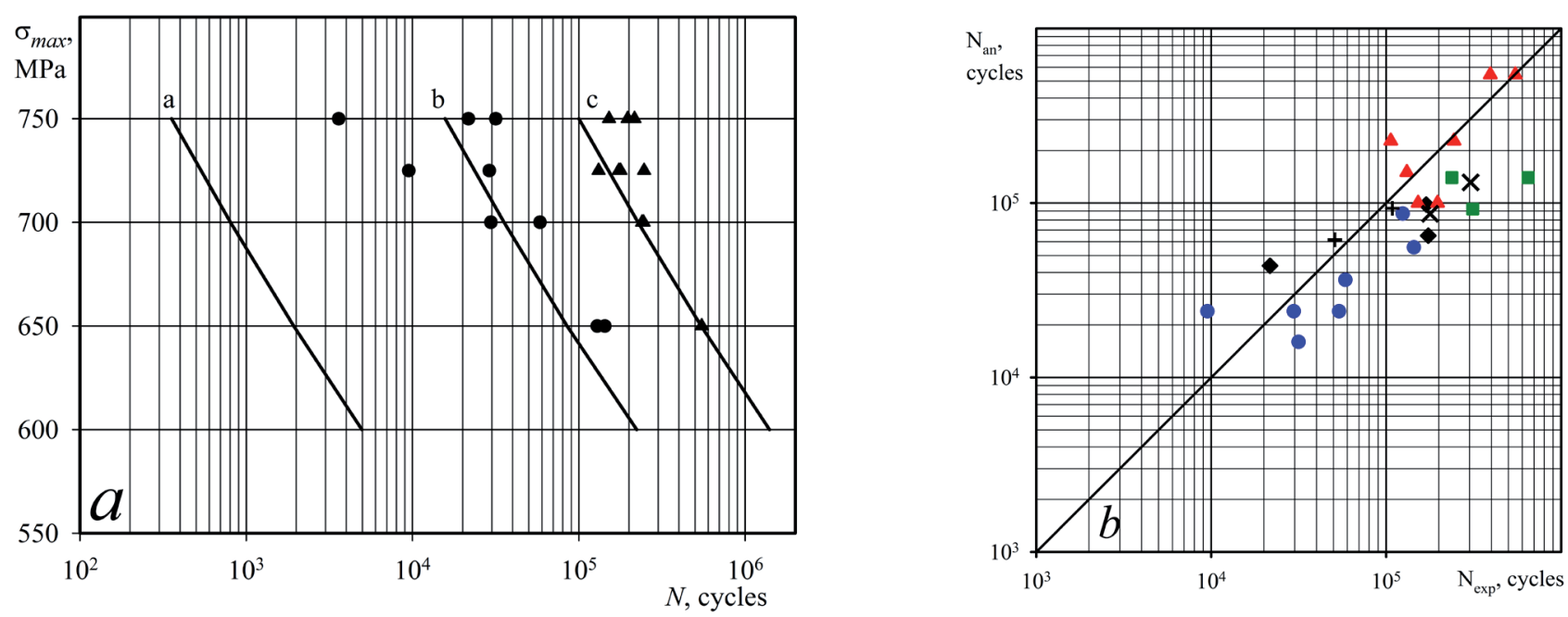

Fig. 3. 3a - Fatigue curves for AISI 5140 under CAL (curve a) and generated spectrums (b - spectra A, c - spectra C); 3b - Correlation of analytical and

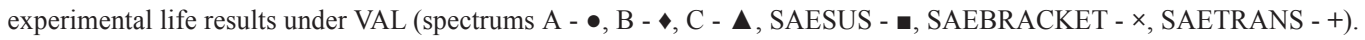

Tab. 3. Nonstationarity factors of spectrum loading for fatigue life evaluation

\begin{tabular}{cccc}
\hline Damage model & Linear & Corrected linear & Based on spectrum character \\
\hline $\begin{array}{c}\text { Nonstationarity } \\
\text { factor }\end{array}$ & $K_{1}=\frac{1}{V^{m}}$ & $K_{2}=\frac{a_{p}}{V^{m}}$ & $K_{3}=10^{(1+q \lg m)(1-V)}$ \\
\hline
\end{tabular}

Tab. 4. AISI 5140 fatigue life prediction by various damage models and under several loading cases

\begin{tabular}{|c|c|c|c|c|c|c|c|c|}
\hline \multirow{2}{*}{ Loading spectra } & \multirow{2}{*}{$\begin{array}{l}\text { Spectrum } \\
\text { fullness factor } V\end{array}$} & \multicolumn{3}{|c|}{$\begin{array}{c}\text { Nonstationarity factor by } \\
\text { various models }\end{array}$} & \multirow[t]{2}{*}{$\begin{array}{l}\text { Value of } N_{\Sigma \text { exp }}, \\
\text { cycles }\end{array}$} & \multicolumn{3}{|c|}{ Durability ratio $N_{\Sigma i} / N_{\Sigma \exp }$ for models } \\
\hline & & $\mathrm{K}_{1}$ & $\mathrm{~K}_{2}$ & $\mathrm{~K}_{3}$ & & 1 & 2 & 3 \\
\hline CAL & 1 & 1 & 1 & 1 & 2000 & - & - & - \\
\hline Spectra A & 0.676 & 101.5 & 31.5 & 45 & $1.3 \cdot 10^{5}$ & 1.56 & 0.49 & 0.69 \\
\hline Spectra C & 0.52 & 936 & 137 & 282 & $5.5 \cdot 105$ & 3.40 & 0.50 & 1.03 \\
\hline
\end{tabular}

Simplicity and minimal set of structural-sensitive parameters are typical advantages of the proposed models. As stated above, generalized model considers material properties and loading character and consequently exhibits the most adequate results. Linear model has sufficient use in commercial software, though it shows overoptimistic results. It is caused by criterional damage parameter $D(0 \leq D \leq 1)$ that demonstrates huge divergence associated with loading parameters.

Conspicuously that criteria parameter of corrected linear model alters within the range $0 \leq D \leq a_{p}$, where $a_{p}<1$. According to expectations, this model shows more adequate results than linear, but, to the contrary, it usually underestimates strength. Correlation of calculated life $N_{a n}$ by formula (4) and experimental result $N_{\text {exp }}$ of steel AISI 5140 under different VAL spectrums is illustrated in fig. 3b. One can notice good correlation of these data. Let us mark that correlation factor is $\vartheta=0.817$, and root mean square is $S_{\sigma}=0.742$.
In such a manner, loading character of VAL has a substantial effect on fatigue life of steel AISI 5140. The character stands on spectrum fullness factor $V$ and cyclic strength properties under CAL.

\section{Inelasticity and fatigue life relationship under VAL}

Evolution of inelastic processes in metals under cycling is directly related to propagation of micro-plastic strains [13, 14]. These strains define kinetics of fatigue damage cumulation and lead to fracture. Therefore evolutional kinetics of hysteresis loops was analyzed. A set of loops of one our spectrum is plotted in stress against strain coordinates and distinct hysteresis loops were extracted (fig. 4).

Signals from extensometer and load cell were recording during test series. Let us remark that 20 measuring points represented both ascending and descending reversals. This data assured smooth approximation of loops with minimal 
accuracy $10^{-5}$. Width loop evolution during applied narrowband normalized spectrums $\mathrm{A}(a)$ and $\mathrm{C}(b)$ with maximal stress value $\sigma_{a \max }=700 \mathrm{MPa}$ is plotted in fig. 5 . One can observe that strain leaps caused by extreme stress values are more abundant for spectrum A than for C. Gradual increasing of width loop accompanied by both maximal stress and mean stress growth for spectrum A.

Fragment of micro-plastic strain cumulation and effect of stress amplitude altering on it are shown in fig. 6a for the spectrum A $\left(\sigma_{a \max }=725 \mathrm{MPa}\right)$. Interrelation of inelasticity parameters and life is shown for CAL (curve $a$ ) and VAL (curves $b, c$ ) in fig. 6b. They were plotted with using of life values based on Coffin-Manson equation in "Stoflo" program [15].

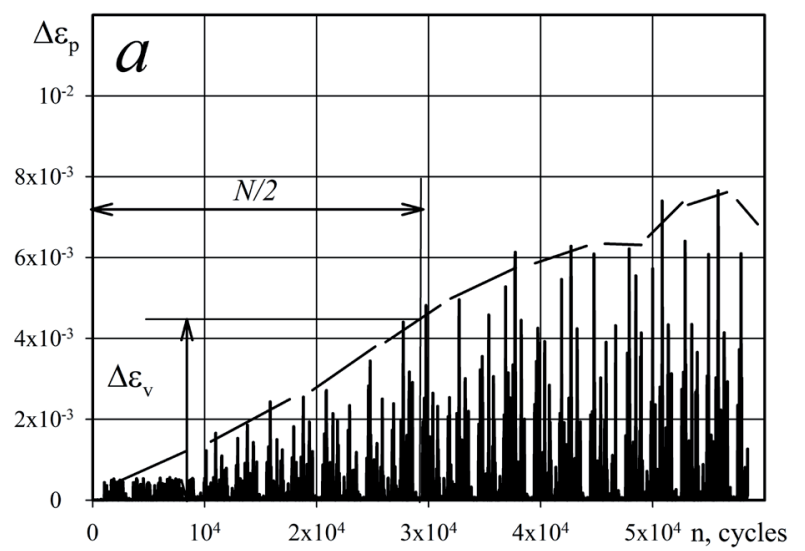

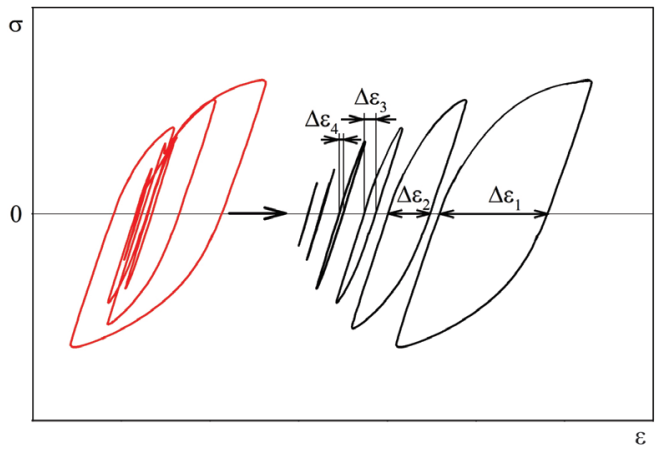

Fig. 4. Accounting of hysteresis loops evolution by the example of a recorded fragment of inelastic processes kinetics under VAL

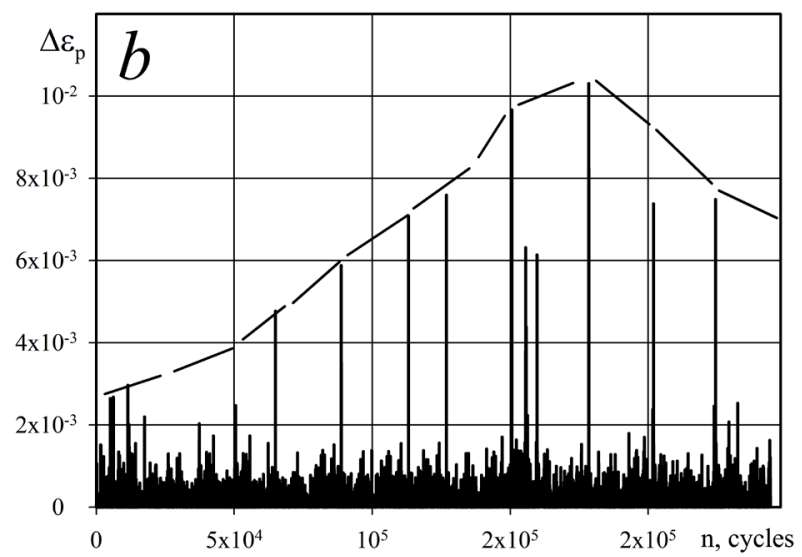

Fig. 5. The evolution of hysteresis loop under various spectrums with $\sigma_{a \max }=700 \mathrm{MPa}(a-\operatorname{spectra} \mathrm{A}, b-\operatorname{spectra} \mathrm{C})$
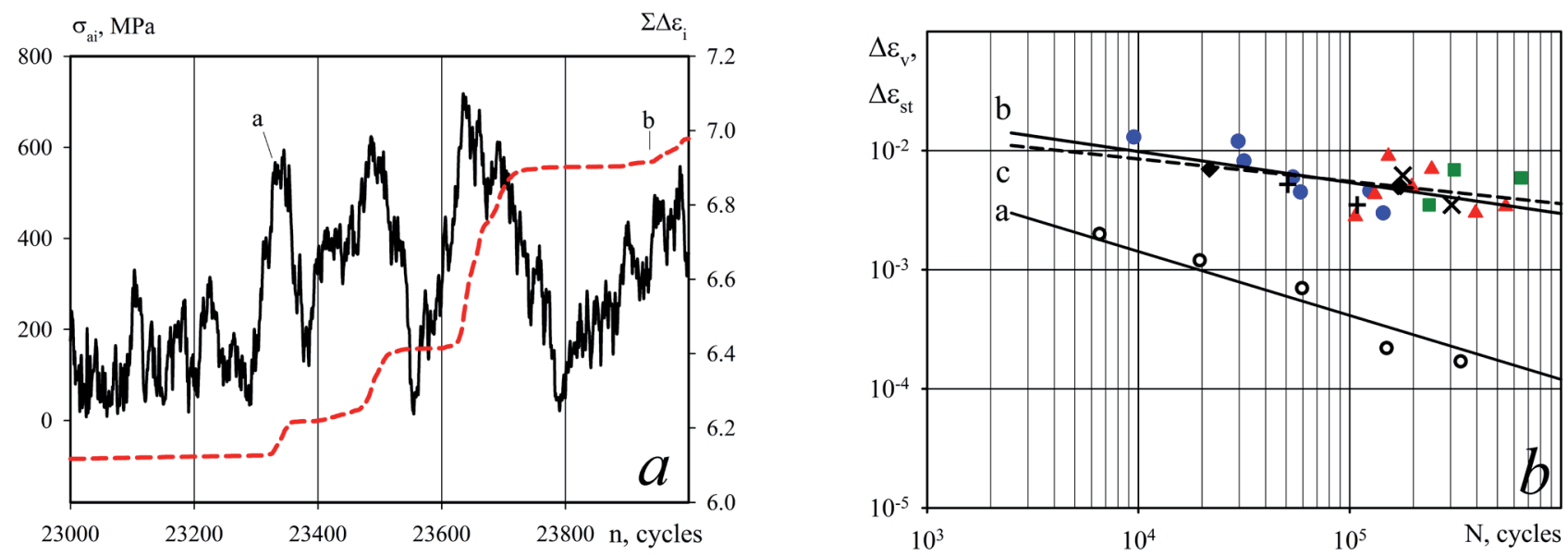

Fig. 6. 6a-Fragments of maximal stress envelop curve (a) and micro-plasticity cumulation curve (b) at an exemplary region of VAL (spectrum A); $6 \mathrm{~b}-$ Plot of inelastic strains $\Delta \varepsilon_{v}, \Delta \varepsilon_{s t}$ against fatigue life $N$ for AISI 5140 (o-CAL, $\bullet-$ spectrums A, $\left.\Delta-\mathrm{C}, \bullet-\mathrm{B}, \boldsymbol{\bullet}-\mathrm{SAESUS}, \times-\mathrm{SAEBRACKET},+-\mathrm{SAETRANS}\right)$

Features of inelastic processes were compared under CAL and VAL. In case of CAL relationship between inelasticity and material durability was established on the basis of loop width approach that assumes correspondence between loop width $\Delta \varepsilon_{s t}$ and half durability life to fracture $N / 2$ for current stress amplitude $\sigma_{a}=\sigma_{a \max }$ (fig. 6b, curve $a$ ). Obviously, it is required to select criterional parameter to clarify relationship between inelastic strains and durability life under VAL. Loop width evolution was evaluated for $\sigma_{a}=\sigma_{a \max }$ that relevant to half durability life to fracture, so it is advisably to correlate 
criterional parameter $\Delta \varepsilon_{p}$ with $\sigma_{a}=\sigma_{a \max }$ (fig. 5a). In case of same values $\Delta \varepsilon_{s t}=\Delta \varepsilon_{p}$ under VAL spectrum fullness factor for VAL is smaller $V(1)$ than for CAL $(V=1)$, so durability life for VAL is longer than for CAL according to formula (4). Thus same durability life for CAL and VAL implies that value $\sigma_{a \max }$ is higher for VAL than for CAL (fig. 6b, curve $b$ ).

\section{Steel damage simulation under VAL by strain and energy criterions}

Fatigue fracture usually initiates in regions of local stress concentration (stress raisers). Variable micro-stresses lead to micro-plastic strain cumulation and finally crack appears if plasticity is fully exhausted in overstrained volumes. Unfortunately, direct measurement of damage cumulation in local volumes is difficult. Therefore engineers use local stress-strain approach for fatigue life estimation of structural parts under cyclic loading to avoid this lack. This method gives an advantage to predict durability life of variety of structures, to complete analysis with using of specimens test data, including their behavior with interaction effects. Local approach admits to calculate durability life based on kinetics of hysteresis loops evolution that corresponds to damage cumulation in metal. This approach can be used in issues if plastic strain amplitude has the same or higher value at both critical point and local stress raiser. Fatigue resistance evaluation arises according to relationship between stress and strain based loops generated by CAL. Cyclic stress-strain curve based on hysteresis loop evolution is used in this approach for fatigue resistance estimation. Expression for the cyclic curve is shown below:

$$
\frac{\Delta \varepsilon}{2}=\frac{\Delta \varepsilon_{e}}{2}+\frac{\Delta \varepsilon_{p}}{2}
$$

where $\Delta \varepsilon, \Delta \varepsilon_{e}, \Delta \varepsilon_{p}-$ total, elastic and plastic strain components of hysteresis loop correspondingly.

Elastic strain component can be evaluated by expression:

$$
\frac{\Delta \varepsilon_{e}}{2}=\frac{\Delta \sigma}{2 E}
$$

where $E$ - elastic modulus.

Plastic strain component can be defined by formula:

$$
\frac{\Delta \varepsilon_{p}}{2}=\left(\frac{\Delta \sigma}{2 K^{\prime}}\right)^{\frac{1}{n^{\prime}}}
$$

where $K^{\prime}$ - constant; $n^{\prime}$ - coefficient of cyclic hardening (softening).

Hence, cyclic strain curve corresponds to equation:

$$
\frac{\Delta \varepsilon}{2}=\frac{\Delta \sigma}{2 E}+\left(\frac{\Delta \sigma}{2 K^{\prime}}\right)^{\frac{1}{n^{\prime}}}
$$

Fatigue curve equation is all-known:

$$
\Delta \sigma=C \cdot N^{B}
$$

In the end, Coffin-Manson equation is received [14]:

$$
\frac{\Delta \varepsilon}{2}=\frac{\sigma_{f}^{\prime}}{E}\left(2 N_{f}\right)^{b}+\varepsilon_{f}^{\prime}\left(2 N_{f}\right)^{c},
$$

where $\sigma_{f}{ }^{\prime}$ - true tensile strength; $\varepsilon_{f}{ }^{\prime}$ - true plastic strain; $b, c$ - material constants; $2 N_{f}=N$ - number of cycles to fracture.

Various generated spectrums (table 2) are used for fatigue life calculations. Original material data for AISI 5140 are applied for analysis in "Stoflo" program (table 1).

Fatigue curve (3) in fig. 6b is obtained by local strain-stress approach. It is evident that experimental values coincide to the Coffin-Manson curve. Experimental results (dots in fig. 5) are independent of loading character and rely on plastic strain, so one can see that experimental and analytical results correlate for various spectrums. Damage was estimated by energy criterions under VAL for steel AISI 5140. These calculations are based on plasticity exhaustion $d_{p}$ and dissipation of elastic energy in material defined by hysteresis loop variation $d_{e}$.

$$
d_{\Sigma}=d_{p}+d_{e}
$$

Plastic component of damage $d_{p}$ is evaluated by energy criterion:

$$
d_{p}=\sum_{i=1}^{N} \frac{D_{p i}}{D_{p i}^{c r}}
$$

where $D_{p i}-$ dissipated energy per cycle of VAL:

$$
D_{p i}=k_{f} \Delta \varepsilon_{i} \sigma_{a i}
$$

where $k_{f}, \Delta \varepsilon_{i}, \sigma_{a i}$ - parameters of hysteresis loop.

Critical energy value corresponding to fracture is defined by expression [16]:

$$
D_{p i}^{c r}=s_{u}\left(\frac{\sigma_{p}}{\sigma_{a i}}\right)^{\Theta}
$$

where fracture energy per volume $s_{u}=830 \mathrm{MN} / \mathrm{M}^{2}$, true tensile strength $\sigma_{p}=1436 \mathrm{MPa} ; \Theta=2.85$ - material constant.

Apparently, longer fatigue life $N$ corresponds to higher $D_{p i}^{c r}$ and lower $\sigma_{a i}$ values.

Elastic part of damage can be estimated by formula:

$$
d_{e}=\sum_{i=1}^{N} \frac{D_{e i}}{D_{e}^{c r}}
$$


where elastic component of dissipated energy per deformation cycle is as follows:

$$
D_{e i}=\frac{\Delta \sigma_{i} \Delta \varepsilon_{i}}{2}=\frac{2 \sigma_{a i}^{2}}{E}
$$

where $\Delta \sigma, \Delta \varepsilon-$ stress and strain range per cycle.

Critical elastic component of dissipated energy depends on fatigue endurance limit $\sigma_{-1}$ and for knee point on fatigue curve $N_{G}$ :

$$
D_{e}^{c r}=\frac{2 \sigma_{-1}^{2} N_{G}}{E}
$$

Finally damage estimation by elastic component was received:

$$
d_{e}=\frac{\sum_{i=1}^{N} \sigma_{a i}^{2}}{\sigma_{-1}^{2} N_{0}}
$$

It is well-known that damage variation depends on durability to fracture. Mixed mode of fracture considers both plastic and elastic damage cumulation components. Elastic component attributed with fatigue damage cumulation and, consequently, initiation and propagation of crack. By default, fracture occurs if $d_{\Sigma}=1$. Analytical fracture surfaces are shown for total damage $d_{\Sigma}$ based on proposed damage expressions in fig. 7: $1-d_{\Sigma}=d_{p l}+d_{e l}$ is for linear model of damage; $2-d_{\Sigma}=d_{p l}^{\alpha}+d_{e l}^{\beta}$ is for nonlinear model of damage and damage components interaction. In this model nonlinear damage $d_{\Sigma}$ has decelerated character of plastic component $d_{p l}(\alpha=0.05)$ and accelerated character of elastic component $d_{e l}(\beta=2)$ corresponding to kinetics of fatigue crack propagation.

Total damage values $d_{\Sigma}$ are plotted against number of cycles in fig. 8 by various models under different spectrums for AISI 5140. Plasticity exhaustion model (fig. 8a) shows that total damage value $d_{\Sigma}=0.56$ (mean square deviation $s_{d}=0.324$ ) is lower than in suggested criterion. Linear damage model of mixed fracture mechanism (fig. 8b) approximates damage value to criterional one $d_{\Sigma}=0.7\left(s_{d}=0.332\right)$. However, sufficient scatter of damage values for various spectrums and loading conditions is typical for this model. Nonlinear damage model (fig. 8c) demonstrates decelerated character of plastic component $d_{p l}(\alpha=0.05)$ and accelerated character of elastic component $d_{e l}(\beta=2)$ and fits experimental results to criterional parameter value $d_{\Sigma}=0.991\left(s_{d}=0.039\right)$.

\section{Discussion}

Presented research of steel durability estimation under random loading showed that character of loading (represented by spectrum fullness factor) has a significant effect on fatigue life. Durability analysis based on power criterions clearly distinguishes fatigue curves under VAL with maximal stress $\sigma_{a \max }($ fig. 3). If current stress exceeds yield point, then extensive scatter emerges in durability life for same values $\sigma_{a \max }$. Probably, it is related to plastic strains interaction under random loading. Damage models have phenomenological or halfphenomenological character and based on experimental results. The most significant scatter between analytical and test results are inherent to linear model. Conversely, model considering loading character (table 5) reveals fine correlation between analogue results. Durability estimation by strain criterion via Coffin-Manson model discovered that it determines durability more precisely despite on loading type. However even strain criterion tends to have a statistical scatter between experimental and analytical results. Defined curve $c$ is close to curve $b$ (fig. 6b). Notable, experimental data lie strictly along curve $b$ for all spectrums. Though little slope of curve $b$ in coordinates $\lg \Delta \varepsilon-\lg N$ causes huge durability sensitivity to $\Delta \varepsilon_{p}$. Reciprocally, negligible drawback of $\Delta \varepsilon_{p}$ leads to unacceptable mistake in durability value up to 10 times.

Energetical approach for durability calculation was completed via linear and nonlinear damage cumulation models related to plastic and elastic strain components. Good
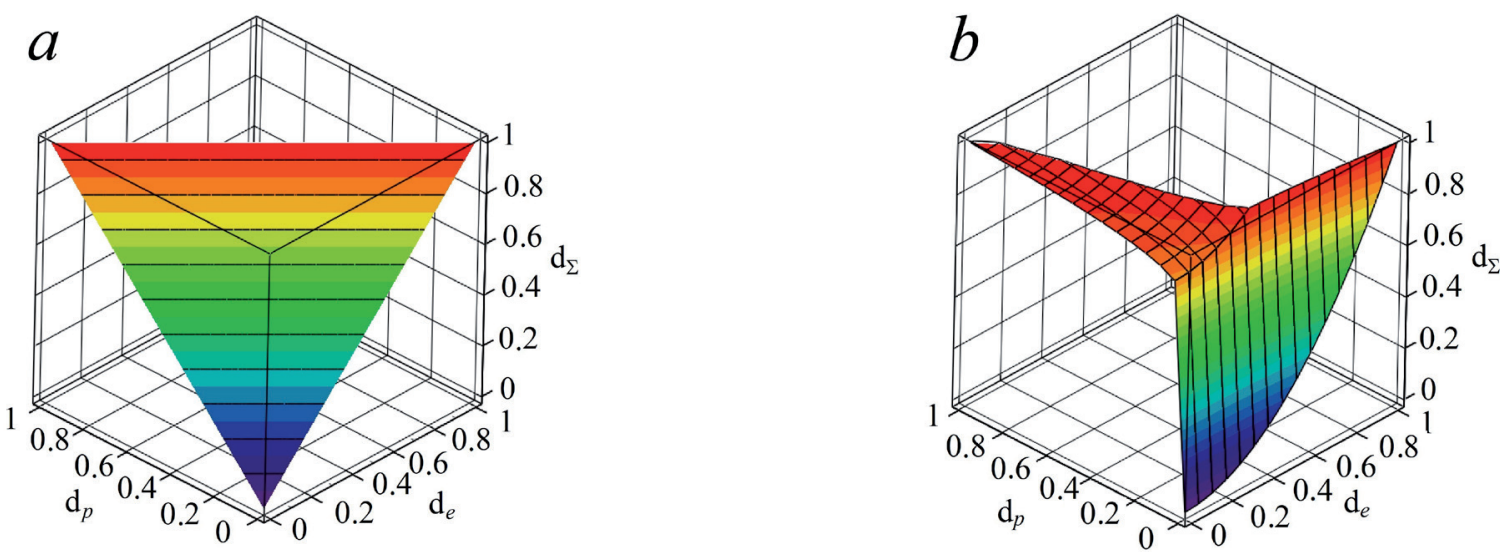

Fig. 7. Analytical fracture surfaces for cumulation of elastic $d_{e}$ and plastic $d_{p}$ damage components by linear $(a)$ and nonlinear $(b)$ models. 

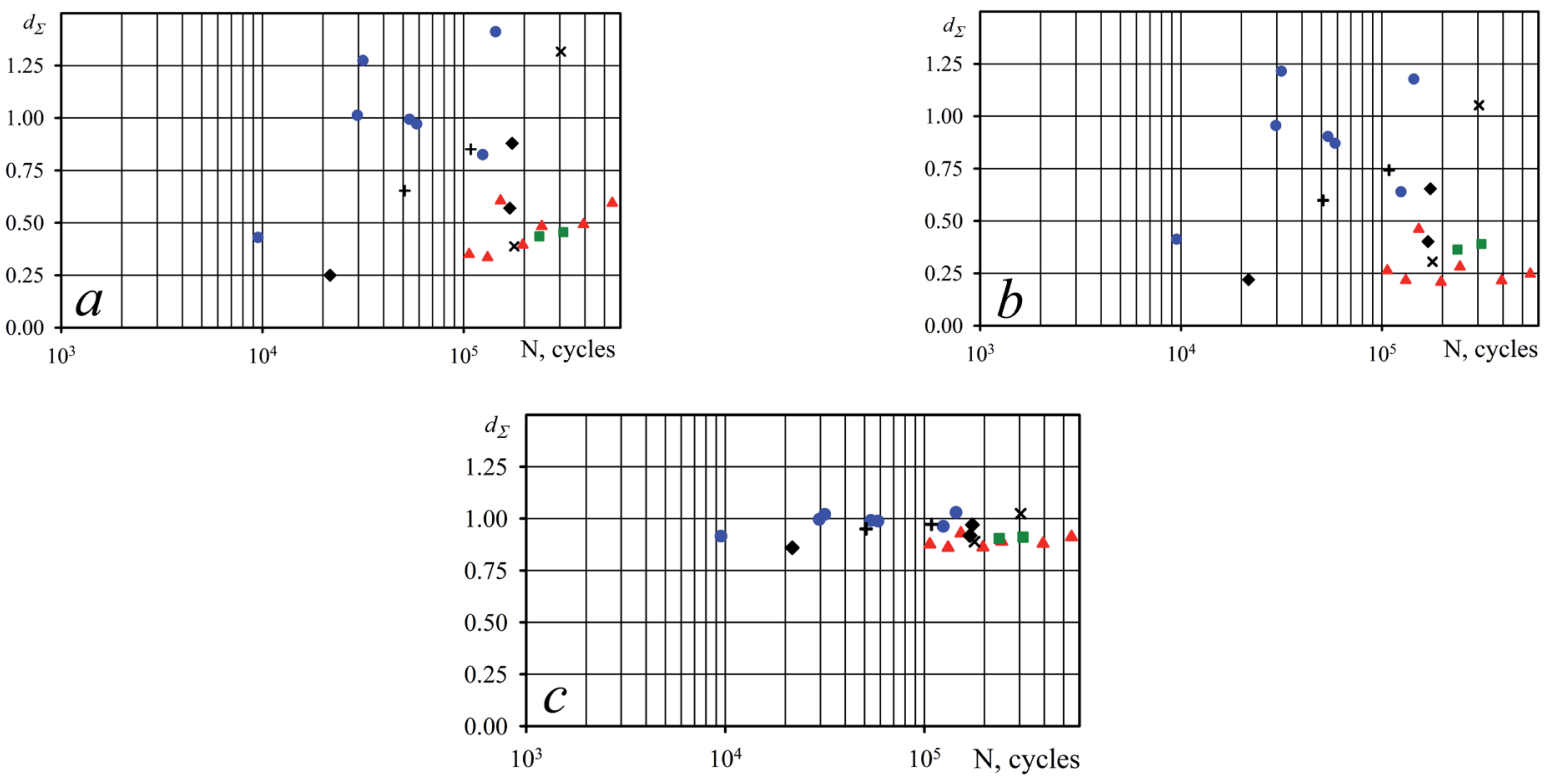

Fig. 8. Calculated damage value $d_{\Sigma}$ related to various damage models and spectrum loading: $\mathrm{a}-d_{\Sigma}=d_{p l}$ - by plasticity exhaustion model; $\mathrm{b}-d_{\Sigma}=d_{p l}+d_{e l}-$ by linear damage model in mixed fracture; $\mathrm{c}-d_{\Sigma}=d_{p l}^{\alpha}+d_{e l}^{\beta}$ - by nonlinear damage model in mixed fracture

correlation between experimental and analytical results can be discovered by means of nonlinear damage cumulation model considering interaction of fracture mechanisms.

\section{Summary}

Evaluation of durability life showed that presented damage models and criterions can be successfully used in engineering practice for AISI 5140 under random loading. Power approach offers approximate durability calculation using minimal set of input material properties and loading data. More precise and physically conditioned results can be obtained by strain and energetical approaches, but analysis is restricted by range $0,002<\Delta \varepsilon_{p} / 2<0,02$ and it is impossible to expand this region to short and very long forecasts of durability life. In further, additional research work is required to consider stress concentration and fracture mechanics concept for durability estimation of structural parts.

\section{References}

1 Kwofie S., Rahbar N., A fatigue driving stress approach to damage and life prediction under variable amplitude loading. International Journal of Damage Mechanics, 22 (3), pp. 393-404, (2013). DOI: $\underline{10.1177 / 1056789512449638}$

2 Macha E., Lagoda T., Nieslony A., Kardas D., Fatigue life under variable-amplitude loading according to the cycle-counting and spectral methods. Materials Science, 42 (3), pp. 416-424, (2006). DOI: 10.1007/s11003-006-0097-2

3 Djeblia A., Aid A., Bendouba M., Amrouche A., Benguediab M., Benseddiq N., A non-linear energy model of fatigue damage accumulation and its verification for Al-2024 aluminum alloy. International Journal of Non-linear Mechanics, 51, pp. 145-151, (2013). DOI: 10.1016/j.ijnonlinmec.2013.01.007

4 Median Test System (2014). http://www.biss.net.in/median-test-system.htm

5 GOST 25.502-79, Strength Analysis and Testing in Machine Building. Methods of Metals Mechanical Testing. Methods of Fatigue Testing. M.: Publisher of Standards, p. 25, (2005).

6 Bannantine J. A., Comer J. J., Handrock J. L., Fundamentals of Metal Fatigue Analysis. Prentice Hall, New Jersey, (1990).

7 GOST 25.507-85, Strength Calculation and Testing in Machine Building. Methods of Fatigue Testing under Service Loading. General Requirements. M.: Publisher of Standards, p. 19, (2005).

8 Sarkani S., Feasibility of Auto-Regressive Simulation Model for Fatigue Studies. Journal of Structural Engineering, 116 (9), pp. 24812495, (1990). DOI: $\underline{\text { 10.1061/(ASCE)0733-9445(1990)116:9(2481) }}$

9 Kihl D. P., Stochastic Fatigue Concepts in Welded Surface Ship Structures. Departmental Report SSPD-90-173-25, US Navy: David Taylor Research Center, Bethesda, MD 200084-5000, (1990).

10 Miner M. A., Cumulative Damage in Fatigue. Journal of Applied Mechanics, Vol. 67., pp. A159-A164, (1945).

11 Kogaev V. P., Makhutov N. A., Gusenkov A. P., Strength and Durability Analysis of Machine Parts and Structures: Handbook. Machinery, Moscow, (1985).

12 Savkin A. N., Bagmutov V. P., Fatigue Durability Estimation of Heavy-duty Structures. VSTU, Volgograd, (2013).

13 Schijve J., Fatigue of Structures and Materials. Springer, (2008)

14 Manson S. S., Fatigue: A complex subject-Some simple approximations. Experimental Mechanics, 5 (4), pp. 193-226, (1965). DOI: $10.1007 / \mathrm{BF} 02321056$

15 Rainflow Cycle, Counting Excel Template with Macros (2011). http://www.storera.com/stoflo/

16 Troschenko V. T., Metal Straining and Fracture under Multicycle Loading. Kiev: Naukova Dumka, (1981). 